\title{
A reevaluation of the petrogenetic relationships among HED meteorites with $\mathrm{Ca}$ isotopes
}

\author{
Maria C. VALDES ${ }^{*}$, Clara L. BlätTLER ${ }^{2}$, AND PHILIPP \\ R. HECK ${ }^{12}$
}

'Robert A. Pritzker Center for Meteoritics and Polar Studies, The Field Museum of Natural History, Chicago, IL, USA. Department of The Geophysical Sciences, The University of Chicago, Chicago, IL, USA.

The idea that howardite, eucrite, and diogenite (HED) meteorites are derived from a single parent body, Asteroid 4 -Vesta, is primarily based on oxygen (O) isotope data, which define a homogenous, linear array in a three-isotope diagram that lies below the terrestrial fractionation line [e.g. 1]. HEDs are assumed to be related to each other by the igneous differentiation of a global Vestan magma ocean; as such, they offer a chance to understand this early and possibly widespread type of activity in the early solar system. However, more recent $\mathrm{O}$ isotope measurements have revealed deviations of some eucrites and howardites from the common mass fractionation line [2]. This, along with the enduring lack of a magma ocean crystallization model that explains petrologic observations, suggests that Vesta experienced incomplete homogenization during primary differentiation or subsequent partial melting of its silicate mantle [2-3]. Alternatively, it may be that HEDs do not share a common parent body or that carbonaceous chondritic (CC) admixture into the Vesta regolith plays a role in the chemical signatures of HEDs [2].

This project uses calcium $(\mathrm{Ca})$ isotopes to investigate the nature of HED relationships. Recent studies have begun to evaluate $\mathrm{Ca}$ isotope fractionation related to partial melting of and fractional crystallization in the terrestrial mantle [e.g. 4-5] and results suggest that these processes are sources of mass-dependent $\mathrm{Ca}$ isotope heterogeneity in the products of differentiated bodies. Further quantification of the effects of differentiation processes on $\mathrm{Ca}$ isotopes will allow us to check whether and to what degree the HED clans are igneously related. Furthermore, it is known that mass-dependent $\mathrm{Ca}$ isotope variations exist between the $\mathrm{CC}$ subgroups [6]; these give us a way to calculate whether, how much, and what type of CC material interacted with Vesta regolith.

Dissolutions of 15 monomict HED samples sourced from the meteorite collection at the Field Museum are already underway. Calcium isotopes will be analyzed with a Neptune XT multicollector ICP-MS following automated chromatographic separation with a Thermo-Dionex ICS6000 at The University of Chicago.

[1] Clayton and Mayeda (1983) EPSL 62, 1-6. [2] Wiechert et al. (2004) Science 294, 345-348. [3] Ghosh and McSween (1998) Icarus 134, 187-206. [4] Kang et al. (2017) EPSL 474, 128-137. [5] Valdes et al. (2019) Chem Geol. 509, 77-91. [6] Valdes et al. (2014) EPSL 394, 135-145. 